\title{
Einfluss von pferdegestützten Coachings auf die Selbstwirksamkeitserwartung
}

\author{
Kathrin Schütz ${ }^{1}$ (D) - Julia Steinhoff ${ }^{2}$
}

Online publiziert: 22. März 2019

(c) Der/die Autor(en) 2019

\section{Zusammenfassung}

Die vorliegende Studie untersucht, inwiefern Pferde im Coaching eingesetzt werden können, um die Selbstwirksamkeitserwartung von Personen zu erhöhen. Dabei gehörten Versuchspersonen $(N=106)$, die zwischen 19 und 55 Jahre alt waren $(M=25,91 ; S D=8,25 ; 71 \%$ weiblich), entweder der Versuchsgruppe an und erhielten ein pferdegestütztes Coaching oder der Kontrollgruppe ohne Coaching. Gemessen wurde die Selbstwirksamkeitserwartung jeweils zu zwei Messzeitpunkten. Zudem wurde untersucht, inwiefern sich die Selbstbewertung innerhalb eines pferdegestützten Coachings durch die selbstreflektierende Methode einer Videoanalyse verändert. Hierzu wurde die Selbstbewertung der Coachingteilnehmer sowohl vor als auch nach einer Videoanalyse erfasst. Die Ergebnisse zeigen, dass sich sowohl die Selbstbewertung als auch die Selbstwirksamkeitserwartung durch ein pferdgestütztes Coaching hoch signifikant positiv veränderten und starke Effekte aufwiesen. Weiterhin konnte ein mittlerer Zusammenhang zwischen der Selbstbewertung und der Selbstwirksamkeitserwartung festgestellt werden. Menschen mit und ohne Pferdeerfahrung unterschieden sich zudem im ersten Messzeitpunkt signifikant voneinander, während sie sich im zweiten Messzeitpunkt eher anglichen. Die Selbstwirksamkeitserwartung stieg bei Menschen ohne Pferdeerfahrung mehr an als bei Menschen mit Pferdeerfahrung. Die Langzeitwirkung der Intervention, weitere Geschlechterunterschiede und die Wirkfaktoren der Videoanalyse gilt es bei dem nach wie vor kaum erforschten Bereich des pferdegestützten Coachings in Folgestudien zu untersuchen.

Schlüsselwörter Selbstwirksamkeitserwartung $\cdot$ Pferdegestütztes Coaching $\cdot$ Videoanalyse $\cdot$ Pferde

Kathrin Schütz

kathrin.schuetz@hs-fresenius.de

1 Psychology School, Hochschule Fresenius University of Applied Sciences, Platz der Ideen 2, 40476 Düsseldorf, Deutschland

2 Psychology School, Hochschule Fresenius University of Applied Sciences, Im MediaPark 4c, 50670 Köln, Deutschland 


\title{
Effects of Horse-Assisted Coachings on the Self-Efficacy Expectation
}

\begin{abstract}
The present study examines whether horses can be implemented in coachings in order to increase individuals' self-efficacy expectation. Therefore, test subjects $(N=106)$, aged 19-55 $(M=25.91, S D=8.25 ; 71 \%$ female), belonged either to the experimental group (taking part in a horse-assisted coaching) or the control group (without any coaching). The self-efficacy expectation was evaluated using two measurement points. Furthermore, effects of self-reflecting video analyses in horse-assisted coachings on the individuals' self-assessment were examined. Therefore, the self-assessment was gathered both before and after the video analyses. The results show that both the self-assessment and the self-efficacy expectation were significantly and positively influenced by a horse-assisted coaching (medium to large effects). Furthermore, a moderate correlation between the self-assessment and the self-efficacy expectation could be identified. Significant differences between persons with and without experience with horses could be found at the first point of measurement, but they became more similar at the second point of measurement. The self-efficacy expectation led to a stronger increase among individuals without experiences with horses than among individuals with experience with horses. The intervention's long-term effect, further gender differences and effects of the video analysis have to investigated in further studies in the field of horse-assisted coachings.
\end{abstract}

Keywords Self-efficacy expectation $\cdot$ Horse-assisted coaching $\cdot$ Video analysis $\cdot$ Horses

\section{Einleitung}

Tiere können in verschiedener Hinsicht positive Wirkungen auf Menschen haben, wobei die Interaktion mit Tieren stresssenkend, emotional öffnend und auch handlungsmotivierend wirken und ein leichtes sowie nachhaltiges Lernen fördern kann (Friesenhahn 2015a; Greiffenhagen und Buck-Werner 2011). Weitere positive Effekte zeigen sich in einer höheren Selbstachtung und gesteigerten Kontrolle, weshalb Tiere auch im Coaching immer mehr Anwendung finden (Friesenhahn 2015a; Greiffenhagen und Buck-Werner 2011; MacDonald 2004; Otterstedt 2001). Obwohl Pferde bereits seit mehreren Jahrzehnten im heilpädagogischen sowie therapeutischen Kontext sowie auch im Coaching immer mehr eingesetzt werden (Friesenhahn 2015a; Gomolla 2014), liegen im Bereich des pferdegestützten Coachings und dessen Wirkung auf Klienten jedoch bislang nur wenige Studien vor (Friesenhahn 2015b).

Betrachtet man das pferdgestützte Coaching, ist es in der Regel die Aufgabe des Klienten verschiedene Übungen mit dem Pferd zu absolvieren, welche anschließend gemeinsam mit dem Coach reflektiert werden (Konir 2012). Dies kann im Setting eines Teamcoachings, Gruppencoachings oder Einzelcoachings stattfinden und je nach Übungszusammenstellung vielfältige Ziele verfolgen (Konir 2012). Die Pferde dienen dabei in einem Coachingsetting als Spiegel und Katalysator für menschliches Verhalten (Meyer 2009). Das generelle Ziel eines Coachings ist es, mittels unterschiedlicher Methoden und Varianten Personen oder auch Gruppen zu einem bestimmten, selbsterwählten Ziel zu bringen (Greif und Rauen 2017). Das Erreichen dieses Ziels beinhaltet oft eine Veränderung in der Einstellung und des Verhaltens des Klienten (Rauen 2014). Als Ausgangspunkt einer differenzierten Selbstreflexion kann dabei das Pferd im Coaching auch eine Verhaltensänderung bewirken (Friesenhahn 2015b).

Um den Bereich des pferdegestützten Coachings im Zusammenhang mit einem psychologischen Konstrukt zu erforschen, welches theoretisch und empirisch fundiert ist, wurde in der vorliegenden Studie die Allgemeine Selbstwirksamkeitserwartung ausgewählt. Dabei handelt es sich um ein Konstrukt, das direkte Auswirkungen auf das menschliche Verhalten haben kann (Bandura 1997), wobei es sich um die subjektive Überzeugung handelt, unbekannte, schwierige Anforderungssituationen auf Grund der eigenen Kompetenzen zu bewältigen (Warner 2017). Seinen Ursprung hat das Konzept der Selbstwirksamkeitserwartung in der sozial-kognitiven Theorie von Bandura (1997) und gilt innerhalb dieser als eine zentrale Komponente, da gemäß dieser Theorie Denken, Motivation, Gefühle und Verhalten durch persönliche Überzeugungen gesteuert werden. Diese Überzeugungen treten beispielsweise als die Ergebnis- und Selbstwirksamkeitserwartung auf (Bandura 1997). Die Ergebniserwartungen beziehen sich auf das Verhalten, welches für das Erreichen eines Ergebnisses notwendig ist (Bandura 1997; Jerusalem 2005). Sie definieren sich also darüber, was eine Person konkret denkt, tun zu müssen, um ein bestimmtes Ergebnis zu erzielen. Abgrenzend hierzu beruht die Selbstwirksamkeitserwartung auf der Überzeugung, das für eine Zielerreichung notwendige Verhalten überhaupt an den Tag legen zu können (Jerusalem 2005). Dieser subjektive Aspekt der individuellen Beurteilung eigener Handlungsmöglichkeiten ist die Kernkomponente der Selbstwirksamkeit (Schwarzer und Jerusalem 2002). Im Wesentlichen lässt sich die Selbstwirksamkeitserwartung nach Bandura (1994) aus vier 
verschiedenen Quellen generieren. Zum einen kann die Selbstwirksamkeitserwartung aus den eigenen Erfahrungen entwickelt werden, wobei Erfolgserfahrungen als das stärkste Mittel gelten, um eine hohe Selbstwirksamkeitserwartung aufzubauen (Bandura 1994). Allerdings muss der Erfolg, sich aus einer schwierigen Lage befreit zu haben, der eigenen Fähigkeit zugeschrieben werden, damit eine Überzeugung der Selbstwirksamkeit entstehen kann. Dem entgegengesetzt können Misserfolge, also eine gescheiterte Bewältigung von Notlagen, zu einer schwächeren Selbstwirksamkeitserwartung führen (Schwarzer und Jerusalem 2002). Ein Ursprung der Selbstwirksamkeitserwartung liegt in der Wahrnehmung eigener Gefühlsregungen (Schwarzer und Jerusalem 2002). Möchte man die selbstbezogene Wirksamkeit erhöhen, so ist es eine Maßnahme, Stressreaktionen zu reduzieren sowie negative emotionale Neigungen und Fehlinterpretationen zu vermeiden. Dabei ist nicht die reine Intensität emotionaler und körperlicher Reaktionen wichtig; vielmehr spielen die Wahrnehmung und Interpretation des Stresses eine zentrale Rolle (Bandura 1994). Eine weitere Quelle der Selbstwirksamkeitserwartung ist die soziale Überzeugung in Form einer verbalen Selbst- und/oder Fremdbeurteilung. Laut Jerusalem (1991) begünstigt eine hohe Selbstwirksamkeit einen situationsunabhängigen Informationsverarbeitungsstil. Dieser kann dafür sorgen, dass angesichts einer anspruchsvollen Aufgabe schneller nach potenziellen Erfolgschancen und dem persönlichen Nutzen Ausschau gehalten wird. Dies kann wiederum mit einer zuversichtlichen Exploration von Lösungsmöglichkeiten einhergehen (Jerusalem 1991). Verfügt eine Person über eine niedrige Selbstwirksamkeit, wird angenommen, dass diese einen eher negativen Verarbeitungsstil verfolgt. Dabei werden außerdem negative Fremdbewertungen besonders fokussiert, folglich als selbstbeschreibend akzeptiert und schließlich für die Selbstbewertung umgesetzt. So können selbstwertbedrohliche Versagensbefürchtungen entstehen (Schunk 1995). In einer Untersuchung von Jerusalem (1990) wurde zudem festgestellt, dass hoch selbstwirksame Personen hohe Stressresistenz zeigten, während Personen mit niedriger Selbstwirksamkeit eher vulnerabel auftraten. Zudem ergab die Untersuchung, dass Menschen mit einer hohen Selbstwirksamkeit ihre Misserfolge external attribuierten, während Menschen mit niedrigerer Selbstwirksamkeit ihr Versagen als Beleg persönlicher Inkompetenz ansahen (Jerusalem 1990). In einer weiteren Studie wurden bei Menschen, deren Selbstwirksamkeit gering ausprägt war, hohe Werte für Angst, Depression, Beschwerden und interpersonelle Probleme gemessen. Konträr dazu wiesen Patienten mit einer hohen Selbstwirksamkeit weniger Angst, Depressionen und körperliche bzw. interpersonelle Probleme auf (Ruholl 2007). Die Selbstwirksamkeitserwartung hat jedoch nicht nur Auswirkungen auf das Innenleben von Personen, sondern auch andere Lebensbereiche und die
Lebensgestaltung können von ihr beeinflusst werden (Göbel und Frese 1999). So fanden Göbel und Frese (1999) eine signifikante, mittlere Korrelation zwischen der Selbstwirksamkeit eines Mitarbeiters und dem Erfolg eines Unternehmens. Weitere Studien weisen auch darauf hin, dass zumindest in einem nicht-akademischen Arbeitskontext ein Zusammenhang zwischen beruflicher Selbstwirksamkeitserwartung und kurz- bis mittelfristigem, objektivem Erfolg besteht (Rosen 2004). Weiterhin konnte festgestellt werden, dass signifikante Korrelationen zwischen der allgemeinen Selbstwirksamkeitserwartung und dreier grundsätzlicher Burn-out-Merkmale (Emotionale Erschöpfung, Zynismus und persönliche Leistungsfähigkeit) bestehen (Frontmüller et al. 2014). Eine Langzeitstudie bei Schülern ergab, dass je geringer die Selbstwirksamkeit ausgeprägt war, desto mehr körperliche Beschwerden wurden empfunden. Zudem schätzten sich höher selbstwirksame Personen als gesünder ein (Satow und Bäßler 1998). Auch konnte festgestellt werden, dass Menschen stärkere Schmerzen empfinden, wenn ihre Selbstwirksamkeit niedriger ist (Boeckle et al. 2016).

Eine Methode, durch die die Selbstwirksamkeit gestärkt werden kann, ist Studien zufolge das Coaching (Baron und Morin 2010; Moen und Allgood 2009; Wakkee et al. 2010). Im Allgemeinen ist Coaching ein Sammelbegriff, unter welchem unterschiedliche Beratungsmethoden zusammengefasst werden. Auch fallen unter den Begriff verschieden Methoden, die Personen oder auch Gruppen zu einem bestimmten Ziel bringen (Greif und Rauen 2017). Beim Coaching handelt es sich also im Wesentlichen um einen Begleitungsprozess, gekennzeichnet durch die individuelle Beratung auf der Prozessebene (Rauen 2005). Der Erfolg eines Coachings kann grundsätzlich mit der Ergebnisqualität beschrieben werden, die sich beispielsweise an der Zielerreichung vorher definierter Ziele sowie an der generellen Zufriedenheit (Heß und Roth 2001) oder anhand des Wohlbefindens des Klienten (Greif 2008) messen lässt. Als Erfolgskriterium wird hier häufig die Beziehung zwischen den Klienten und dem Coach genannt, da es maßgeblich für den Erfolg eines Coachings ist, ob der Klient den Coach sympathisch findet und glaubt, ihm vertrauen zu können (Schreyögg 2003). Doch nicht nur die persönlichen und zwischenmenschlichen Faktoren wirken auf die Ergebnisse des Coachings - auch die Gestaltung des Prozesses sowie die gesamte Struktur kann sich auf den Erfolg auswirken (Heß und Roth 2001). In der Literatur finden sich verschiedene Methoden und Übungen, die in einem Coaching angewendet werden. Ist das Ziel eine Verhaltensänderung seitens des Klienten, kann die Videoanalyse eine zentrale und wirksame Methode sein (FingerHamborg 2005). Die Videoaufzeichnungen des Verhaltens können unter anderem als Beweis für das Verhalten des Klienten dienen und damit seine Selbstreflexionsprozesse aktivieren (Rückle 2005). 


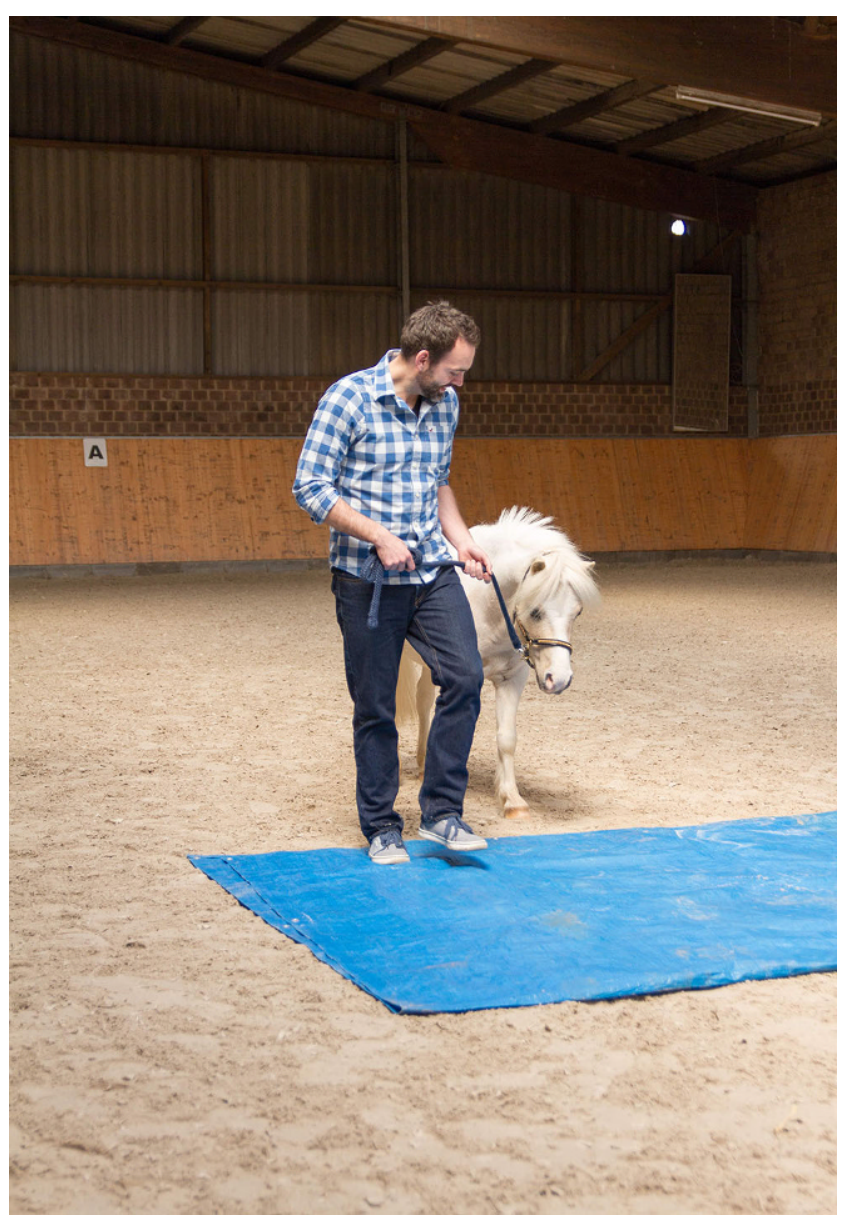

Abb. 1 Beim Überqueren einer knisternden Plastikplane muss das Pferd der führenden Person vertrauen

Die spezielle Coachingform des pferdegestützten Coachings macht sich unter anderem die positive Wirkung einer Mensch-Tier-Beziehung zu Nutze, indem es das Pferd als Coaching-Partner versteht, der sowohl mit dem Coach als auch dem Klienten zusammenarbeitet (Ewing-Chow 2014). Dabei werden dem Klienten Einblicke in die eigene Interaktion mit anderen und seine Fähigkeit, sowohl auf verbaler als auch auf nonverbaler Ebene zu kommunizieren, ermöglicht, wobei unterschiedliche Themen von Einzelpersonen oder Teams bearbeitetet werden (Ewing-Chow 2014; Schütz 2018). Hierbei kann es sich u. a. um die Selbst- und Fremdwahrnehmung, die Wirkung und Ausstrahlung, das Führungsverhalten oder auch die Selbstwirksamkeitserwartung handeln (Schütz 2018). Die Rolle des Pferdes innerhalb des Coachings ist es, dem Klienten als Spiegel seines Verhaltens zu dienen, wobei das Pferd in Übungen vom Boden aus eingesetzt wird (Serad 2010; Schütz 2018). In pferdegestützten Coachings führt man ein Pferd beispielweise im Slalom um Pylonen herum oder über eine Plastikplane (Abb. 1), die für ein Pferd einen ungewohnten Untergrund darstellt. In einer anderen Übung soll der Coachee mit dem
Pferd im Trab über eine auf dem Boden liegende Stange laufen oder er treibt dieses mit der eigenen Körpersprache vor sich her. In einer anderen Aufgabe soll das Pferd dazu gebracht werden, zwischen zwei Stangen rückwärts zu gehen. Pferde gehen lieber vorwärts, da sie dort ihr Umfeld besser im Blick haben. Hier zeigt sich, wie klar kommuniziert wird und ob das Pferd direkt versteht, was es machen soll, stehen bleibt oder in eine andere Richtung geht. Auch das richtige Maß hinsichtlich des Drucks, der benötigt wird, gilt es - wie im Alltag - zu finden und einzusetzen (Schütz 2018).

Zugehörige Videoaufnahmen der Pferd-Mensch-Interaktionen dienen in der nachfolgenden Analyse als Grundlage für den weiteren Coaching-Prozess. Mittels verschiedener Fragetechniken ergibt sich daraus auch die Grundlage für eine intensive Selbstreflexion. Diese ist besonders in der anschließenden, durch einen menschlichen, ausgebildeten Coach geleiteten Reflexion der Übung mit dem Pferd gefragt. Vor dem Hintergrund verschiedener Theorien (z. B. zu Teamrollen, Führung, Kommunikation) werden hierbei die Erfahrungen in der Begegnung mit dem Pferd auf die Berufspraxis oder das Privatleben transferiert, wobei das Pferd dabei als Ausgangspunkt der Selbstreflexion dient. Zusätzlich kann es aber auch als Metapher oder sogar Übungsobjekt für beispielsweise ein klares und souveränes Auftreten dienen (Friesenhahn 2015a; Schütz 2018). Die generelle Wirkung angst- uns stressreduzierende Wirkung von Tieren kann hierbei ebenfalls hilfreich sein (Friedmann et al. 1983; Lang et al. 2010). Weiterhin wurde gezeigt, dass die Anwesenheit von Tieren soziale Interaktionen von Menschen aller Altersstufen fördern kann, wobei der zugrundeliegende Effekt als sozialer Katalysator-Effekt bezeichnet wird (McNicholas und Collis 2000). Damit bewegen die Tiere Menschen dazu, sich emotional zu öffnen und eine Vertrauensbasis zu anderen Mitmenschen aufzubauen, was sich positiv auf die Beziehung zwischen Coach und Klient auswirken kann (Greiffenhagen und Buck-Werner 2011). Besonders Pferde eignen sich hierbei als Coachingpartner, da sie durch Training und Sozialisierung sowie einer vermehrten Interaktion mit dem Menschen in der Lage sind, menschliche Signale feinfühlig zu lesen (Krüger et al. 2011). Dass Pferde mit Menschen interagieren können, zeigte sich auch darin, dass sie in der Lage sind, menschliche Mimik, Gestik und Tonlage zu interpretieren (Nakamura et al. 2018). Zudem reagieren sie auf sowohl Ent- als auch Anspannung ihres menschlichen Gegenübers und spiegeln dessen, zum Teil auch unbewusste, Haltung (Gomolla et al. 2011). Diese Form des Feedbacks kann beim Klienten Lernprozesse anregen, die über Wochen oder Monate nachwirken können (Meyer 2009). Zudem reagieren Pferde in der Kommunikation mit dem Menschen individuell, je nach Situation und dem von dem Menschen ausgehenden Verhalten (Schütz et al. 2018). Da das Konstrukt der Selbstwirksam- 
keitserwartung im Bereich des pferdegestützten Coachings bislang nicht untersucht wurde und somit auch die Wirksamkeit dieser Coachingform analysiert werden kann, wird diese Forschungslücke nachfolgend betrachtet. Dabei soll geprüft werden, ob die Selbstwirksamkeitserwartung überhaupt im Rahmen von pferdegestützten Coachings gesteigert werden kann.

\section{Hypothesen}

Nach Bandura (1994) mobilisieren Menschen, die verbal davon überzeugt sind, Fähigkeiten zu besitzen, eine bestimmte Aktivität zu meistern, mit höherer Wahrscheinlichkeit auch größere Anstrengungen und diese halten diese aufrecht, als wenn sie Selbstzweifel hegen und verbal auf persönliche Defizite eingehen. Wie einflussreich eine hohe Selbstwirksamkeit für ein Individuum sein kann, zeigt sich insbesondere bei der Betrachtung der Folgen, die eine hoch oder niedrig ausgeprägte Selbstwirksamkeitserwartung mit sich bringt. Die Selbstwirksamkeitserwartung lässt sich durch eigene Erfahrungen generieren (Bandura 1994), wobei ein Klient innerhalb eines Coachings die Chance hat, Erfahrungen der Problembewältigung zu machen (Grawe 1998). Zudem kann die Selbstwirksamkeit auch durch stellvertretende Erfahrungen generiert werden (Bandura 1997), was sich auch auf das pferdegestützte Coaching übertragen lässt, in dem das Pferd als Ausgangspunkt der Selbstreflexion dient (Friesenhahn 2015a) und das Tier als Modell für eine stellvertretende Erfahrung fungieren kann. Weiterhin kann die Selbstwirksamkeitserwartung durch die soziale Überzeugung in Form einer verbalen Selbst- und/oder Fremdbeurteilung generiert werden (Bandura 1994). Eine solche Form der Selbst- und Fremdbewertung findet sich auch im pferdegestützten Coaching bei einer gezielten Reflexion der Übungen wieder, bei der die Erfahrungen aus der Begegnung mit dem Pferd auf die Berufspraxis oder das Privatleben transferiert und vor den Hintergrund bewährter Theorien (z.B. zu Teamrollen, Führung, Kommunikation) analysiert werden (Friesenhahn 2015a).

Die aktuelle Studienlage betrachtend zeigt sich, dass man generell von einer Erhöhung der Selbstwirksamkeitserwartung innerhalb eines Coachings ausgehen kann (Baron und Morin 2010; Moen und Allgood 2009). Finn et al. (2007) zeigten zudem, dass sich das Coaching nicht nur direkt positiv auf die Selbstwirksamkeit der Klienten auswirkt, auch die Langzeitwirkung betrachtend, konnte nach sechs Monaten noch eine Erhöhung der Selbstwirksamkeit festgestellt werden. Nicht nur in einem Coachingsetting, auch innerhalb einer pferdegestützten Psychotherapie kann die Selbstwirksamkeitserwartung erhöht werden (Dampsey 2017; Geddes 2010). So gaben beispielsweise bei einer Evaluation einer pferdegestützten, psychologischen Therapie im Anschluss
$37 \%$ der Klienten eine Stärkung der eigenen Selbstwirksamkeit an (Mitteregger 2011). Weiterhin konnte im Rahmen einer klinischen Studie gezeigt werden, dass eine pferdegestützte Therapie die Selbstwirksamkeit im Verlauf von insgesamt acht Sitzungen erhöht (Geddes 2010). Hier liegt die Frage nahe, ob sich ein solcher Effekt auch im Rahmen einer Kurzzeitintervention erzielen lässt.

Da also sowohl eine Steigerung der Selbstwirksamkeitserwartung innerhalb eines Coachings als auch innerhalb einer pferdegestützten Therapie nachgewiesen werden konnte, wurden die folgenden Hypothesen generiert:

H1 Personen, die an einem pferdegestützten Coaching teilgenommen haben, weisen eine signifikant höhere Selbstwirksamkeitserwartung auf als Personen ohne ein solches Coaching.

H2 Personen, die an einem pferdegestützten Coaching teilgenommen haben, weisen nach diesem Coaching eine signifikant höhere Selbstwirksamkeitserwartung auf als vor einem solchen Coaching.

Das Pferd kann als Übungsobjekt für die Bewältigung verschiedener Aufgabe dienen (Friesenhahn 2015a), wobei die erfolgreiche Bewältigung einer Aufgabe ein Faktor zur Steigerung der Selbstwirksamkeit sein kann (Schwarzer und Jerusalem 2002). Jedoch muss das Erfolgserlebnis der eigenen Fähigkeit zugeschrieben werden, um hierdurch die Selbstwirksamkeit zu erhöhen (Schwarzer und Jerusalem 2002). Damit beruht die Selbstwirksamkeit auf Überzeugungen über die eigenen Fähigkeiten also auch auf einer Form der Selbstbewertung. Die Veränderung einer Bewertung der eigenen Fähigkeiten und Leistungen scheint also eine Rolle bei der Veränderung der Selbstwirksamkeitserwartung zu spielen. Die adäquate Untersuchung der Auswirkungen eines pferdegestützten Coachings auf die Selbstwirksamkeitserwartung impliziert daher auch eine Untersuchung der Selbstbewertung. Verfügt eine Person über eine niedrige Selbstwirksamkeit, wird davon ausgegangen, dass sie sich besonders auf negative Fremdbewertungen fokussiert. Diese können als selbstbeschreibend akzeptiert und dann für die Selbstbewertung umgesetzt werden (Schunk 1995). So können negative Fremdbewertungen letztlich in das Selbstkonzept aufgenommen werden und dieses negativ beeinflussen. Eine hohe Selbstwirksamkeit hingegen geht mit einer zuversichtlichen Exploration von Lösungsmöglichkeiten einher (Jerusalem 1991). Daher ist es denkbar, dass eine hohe Selbstwirksamkeitserwartung positive Auswirkungen auf die Leistungsfähigkeit und die Bewertung der eigenen Leistung hat. Eine zentrale Technik innerhalb des Coachings, durch welche auch die eigene Leistung bei der Aufgabenbewältigung reflektiert werden kann, ist die Videoanalyse (Finger-Hamborg 2005). Eine mittels einer Videoanalyse intensivierte Selbstreflexion kann also dazu 


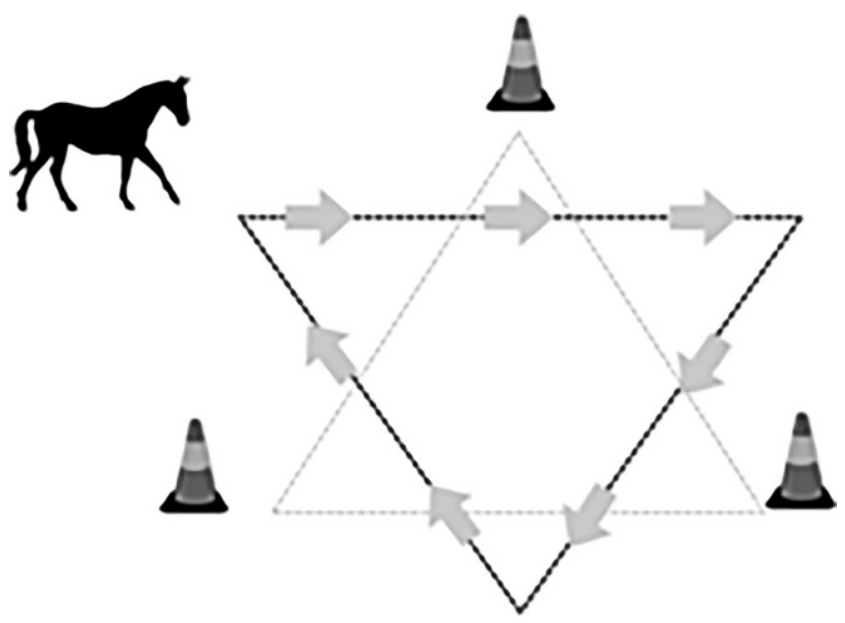

Abb. 2 Aufbau der in der Studie genutzten Übung

führen, das personeneigene Selbstkonzept zu überdenken, abzuwandeln und auch dazu sich positiv zu verändern. Daher lässt sich die nachfolgende Hypothese ableiten:

H3 Personen bewerten sich innerhalb eines pferdegestützten Coachings nach einer Videoanalyse positiver als davor.

\section{Methode}

Die Stichprobe dieser Studie bestand aus insgesamt 106 Versuchspersonen im Alter zwischen 19 und 55 Jahren $(M=25,91 ; S D=8,25$; Angestellte, Selbständige, Studierende), von denen $48 \%(n=51)$ der Experimentalgruppe und $52 \%(n=55)$ der Kontrollgruppe angehörten. $71 \%$ der Personen $(n=75)$ waren weiblich. Des Weiteren gaben $58 \%$ der Teilnehmer an, bereits Erfahrung mit Pferden, z. B. in Form von Reitunterricht, gesammelt zu haben.

Es wurde eine Vergleichsstudie mit Messwiederholung in einem quasi-experimentellen Versuchsdesign durchgeführt (Mc Burney und White 2010). Während die Experimentalgruppe aus Teilnehmern eines eintägigen, pferdegestützten Coachings bestand, setzte sich die Kontrollgruppe aus zufällig randomisierten Personen zusammen. Diese wurden über Aushänge, Internetposts in verschiedenen Gruppen (z.B. facebook) und auf verschiedenen Seiten (z.B. Xing) sowie über persönliche Ansprachen rekrutiert. In der Kontrollgruppe lag die Abbruchquote bei $82 \%$, da diese aktiv an die Teilnahme des zweiten Fragebogens denken mussten. Aus Datenschutzgründen war nicht möglich, Erinnerungs-E-Mails zu versenden.

Das Treatment der Versuchsgruppe erfolgte in Form eines pferdegestützten Coachings. Die Coachings beinhalteten eine Übung, die für die Beantwortung der Fragestellung konzipiert wurde. Hierbei sollten die Probanden im Anschluss an die Kontaktaufnahme eines von zwei Pferden auswählen und eine Übung bewältigen. Die Teilnehmer bekamen, wie in Abb. 2 zu erkennen ist, die Aufgabe, gemeinsam mit dem Pferd zwischen den aufgestellten Pylonen ein Dreieck zu laufen, sodass sich von oben betrachtet ein Stern ergab. Die Übung wurde ausgewählt, da sie einen einfach strukturierten Aufbau beinhaltete und jedem der Pferde aus jeweils mindestens zehn der vorherigen Coachings bekannt war. Weiterhin lag es an den Teilnehmern, wie sie die Aufgabe ausführten; einige vollendeten die Übung mehrfach hintereinander, bei anderen blieb das Pferd an einem Punkt stehen, andere vergaßen, wo sie angefangen hatten. Zudem bot sich so die Möglichkeit, die eigene Leistung zu bewerten, z. B. anhand der Genauigkeit der mit dem Pferd gelaufenen Linien, möglichen Unterbrechungen (Stehenbleiben des Pferdes) und des erneuten Losgehens bzw. Motivierens des Pferdes (wenn es stehen geblieben war).

Bei den in den Coachings eingesetzten Pferden handelte es sich um ausgebildete Pferde (drei Stuten und zwei Wallache) im Alter von 12-20 Jahren, die seit mindestens fünf Jahren in Coachings mitwirkten und die mindestens zehn Stunden pro Tag im Herdenverband stehen. Diese waren darin geschult mit verschiedenen Menschen in Coachings $\mathrm{zu}$ arbeiten.

Insgesamt wurden zwei Fragebögen, jeweils für die EG und die KG, generiert. In beiden Gruppen und Fragebogenteilen wurde die Skala zur Allgemeinen Selbstwirksamkeitserwartung von Schwarzer und Jerusalem (1999) verwendet, wobei es sich um einen Selbstbewertungsfragebogen mit zehn Items zur Erfassung von allgemeinen optimistischen Selbstüberzeugungen handelt. Die Items zielen auf die Frage ab, wie stark eine teilnehmende Person darauf vertraut, die ihr gestellten Herausforderungen meistern zu können. So soll mittels dieser Skala die generelle, optimistische Kompetenzerwartung (Selbstwirksamkeitserwartung) gemessen werden. Weiterhin misst die Skala, ob der Erfolg der eigenen Kompetenz zugeschrieben wird (Schwarzer und Jerusalem 1999). Die theoretische Grundlage der Skala ist das Selbstwirksamkeitskonzept, das von Bandura (1997) als ein wesentlicher Aspekt innerhalb seiner sozial-kognitiven Theorie formuliert wurde. Alle Antwortitems sind vierstufig und gleichsinnig gepolt. Inhaltlich betrachtet bringt jedes Item eine internal-stabile Attribution der Erfolgserwartung zum Ausdruck (Schwarzer 1994). Im Hinblick auf die Gütekriterien der Skala liegen die internen Konsistenzen (Cronbach's Alpha) laut Untersuchungen von Schwarzer und Jerusalem (1999) sowie Schwarzer, Mueller und Greenglass (1999) beim Vergleich von insgesamt 23 Nationen zwischen $\alpha=0,76$ und $\alpha=0,90$. Die Berechnung des Cronbach's Alphas innerhalb dieser Stichprobe ergab bei der Erstmessung einen Wert von $\alpha=0,82$ und bei der Zweitmessung einen Wert von $\alpha=0,89$. Die kriterumsbezogenen Validität konnte durch viele Korrelationsbefunde mit anderen Variablen, wie beispielsweise enge positive $\mathrm{Zu}$ - 
sammenhänge mit dispostionalem Optimismus und negative Zusammenhänge mit Ängstlichkeit und Depression, aufgezeigt werden (Schwarzer und Jerusalem 1999). Weiterhin liegen zu dieser Skala Normwerte vor, die anhand bevölkerungsrepräsentativer Stichproben erhoben wurden (Hinz et al. 2006).

Neben der Skala zur Allgemeinen Selbstwirksamkeitserwartung wurden die Teilnehmer der experimentellen Coachinggruppe gebeten, sich selbst zu beurteilen. Diese Beurteilung fand sowohl direkt nach Beendigung der Aufgabe mit dem Pferd, als auch nach der Videoanalyse, d.h. am Ende des Coachings, statt. Für die Erfassung dieser Selbstbewertung wurde eine Skala mit vier Items generiert, in der unter anderem erfragt wurde, wie korrekt die Übung ausgeführt oder wie gut die Zusammenarbeit mit dem Pferd empfunden wurde. Die Einschätzung dieser Items erfolgte auf einer zehnstufigen Antwortskala von sehr schlecht (1) bis sehr gut (10). Die Teilnehmer konnten eine minimale Punktzahl von 4 und eine maximale Punktzahl von 40 erreichen. Hierdurch sollte ein Bezug zu der Übung mit dem Pferd hergestellt werden, um zu analysieren, ob die Teilnehmenden ihre Leistung im Verlauf des Coachigns unterschiedlich bewerten. Die Reliabilitätsprüfung der selbstgenerierten Selbsteinschätzungsskala ergab eine interne Konsistenz (Cronbach's Alpha) von $\alpha=0,81$.

Beide Gruppen füllten die Fragebögen im Abstand von einer Woche aus, wobei die Experimentalgruppe den ersten Fragebogen eine Woche vor dem jeweiligen Coaching bearbeitete. Im Rahmen der pferdegestützten Coachings, die jeweils durch zwei pferdegestützte Coaches (mit psychologischem sowie medizinischen Hintergrund) sowie einer Assistentin durchgeführt wurden, erfolgte zunächst eine Sicherheitsunterweisung im Umgang mit den Pferden. Die Anliegen der Klienten bezogen sich auf die Selbst- und Fremdwahrnehmung, die eigene Wirkung sowie auf das klare Kommunizieren. Die Übung mit den Pferden wurde im Anschluss durchgeführt, wobei diese ca. ein Drittel des gesamten Coachings einnahm. Jede Durchführung wurde mittels einer Videokamera festgehalten. Direkt im Anschluss an die Übung füllten die Befragten den nur in der Experimentalgruppe vorhandenen zweiten Fragebogen zur Selbsteinschätzung bzgl. der Übung mit den Pferden aus. Danach erfolgten durch die Coaches standardisierte Fragen zur Selbstreflexion der Teilnehmenden, wie diese die Übung wahrnahmen, wie sich diese selbst einschätzten und welche Verhaltensweisen, die sich in der Übung mit dem Pferd zeigten, ihnen aus dem Alltag bekannt vorkamen bzw. neu für sie waren. In der darauf folgenden Videoanalyse wurden die Pferd-Mensch-Interaktionen näher betrachtet und auf die herausgearbeiteten Themen (z.B. eine schlechtere Bewertung der Aufgabe in der Selbstwahrnehmung vs. einer besseren Beurteilung in der Fremdbewertung; unklare Kommunikation, auf die das Pferd in der Aufgabe z.B. durch Stehenbleiben reagiert hatte sowie des Transfers in den Alltag der teilnehmenden Person) eingegangen. Hier wurde einerseits von Seiten der Coaches das Verhalten der Pferde erläutert und andererseits sollten die Teilnehmenden sich selbst beurteilen. Auch hier wurden die eigenen Verhaltensweisen im Hinblick auf den Alltag transferiert. Am Ende des Coachings wurde der abschließende Fragebogen ausgefüllt.

\section{Ergebnisse}

Bei der Betrachtung der deskriptiven Ergebnisse ist zu erkennen, dass die Selbstwirksamkeit zum zweiten Messzeitpunkt in der Experimentalgruppe größer war als in der Kontrollgruppe. Zum anderen zeigte sich, dass die Selbstwirksamkeit in der Experimentalgruppe bei der Zweitmessung größer war als bei der Erstmessung. Die zugehörigen Werte sind in Tab. 1 dargestellt. Bei der Messung der Selbstbewertung innerhalb eines pferdegestützten Coachings konnte vor und nach einer Videoanalyse festgestellt werden, dass die Selbstbewertung vor einer Videoanalyse mit einem Mittelwert von $M=26,39(S D=6,83)$ negativer ausfiel als nach einer Videoanalyse $(M=31,45 ; S D=5,45)$.

Um die Ergebnisse besser veranschaulichen zu können, ist in Abb. 3 ein Balkendiagramm der Mittelwerte der beiden untersuchten Gruppen zu den beiden Haupt-Messzeitpunkten dargestellt.

Die ersten beiden Hypothesen wurden mittels einer univariaten Varianzanalyse mit Messwiederholung (Normalverteilung lag vor) überprüft. Hier zeigte sich ein Interaktionseffekt hinsichtlich der Gruppenzugehörigkeit $(\mathrm{EG} / \mathrm{KG})$, der hoch signifikant war $(F(1,104)=163,42$; $\left.p=0,00 ; \eta_{p}{ }^{2}=0,25\right)$, wie er in der ersten Hypothese postuliert wurde. Dabei handelt es sich um einen starken Effekt. Diese Hypothese kann demzufolge angenommen werden. Die Teilnahme an einem pferdegestützten Coaching ging somit mit einer höheren Selbstwirksamkeitserwartung einher als ohne Teilnahme. Weiterhin zeigte sich ein hoch signifikanter Haupteffekt des Messzeitpunkts $\left(F(1,104)=12,43 ; p=0,00 ; \eta_{p}{ }^{2}=0,11\right)$, welcher in der der zweiten Hypothese postuliert wurde. Hier handelt es sich ebenfalls um einen starken Effekt. Auch diese Hypothese, die eine Steigerung der Selbstwirksamkeitserwartung nach einem pferdegestützten Coaching postulierte, gilt als vorläufig bestätigt.

Tab. 1 Mittelwerte der Selbstwirksamkeitserwartung der EG und KG im Prä-Post-Test-Vergleich

\begin{tabular}{lllll}
\hline & \multicolumn{2}{l}{ Experimentalgruppe } & \multicolumn{2}{l}{ Kontrollgruppe } \\
& $M$ & $S D$ & $M$ & $S D$ \\
\hline Prä-Test & 28,92 & 4,48 & 29,85 & 4,38 \\
Post-Test & 31,75 & 5,24 & 29,16 & 4,31 \\
\hline
\end{tabular}


Abb. 3 Diagramm zum Vergleich der beiden Gruppen im Prä- und Posttest

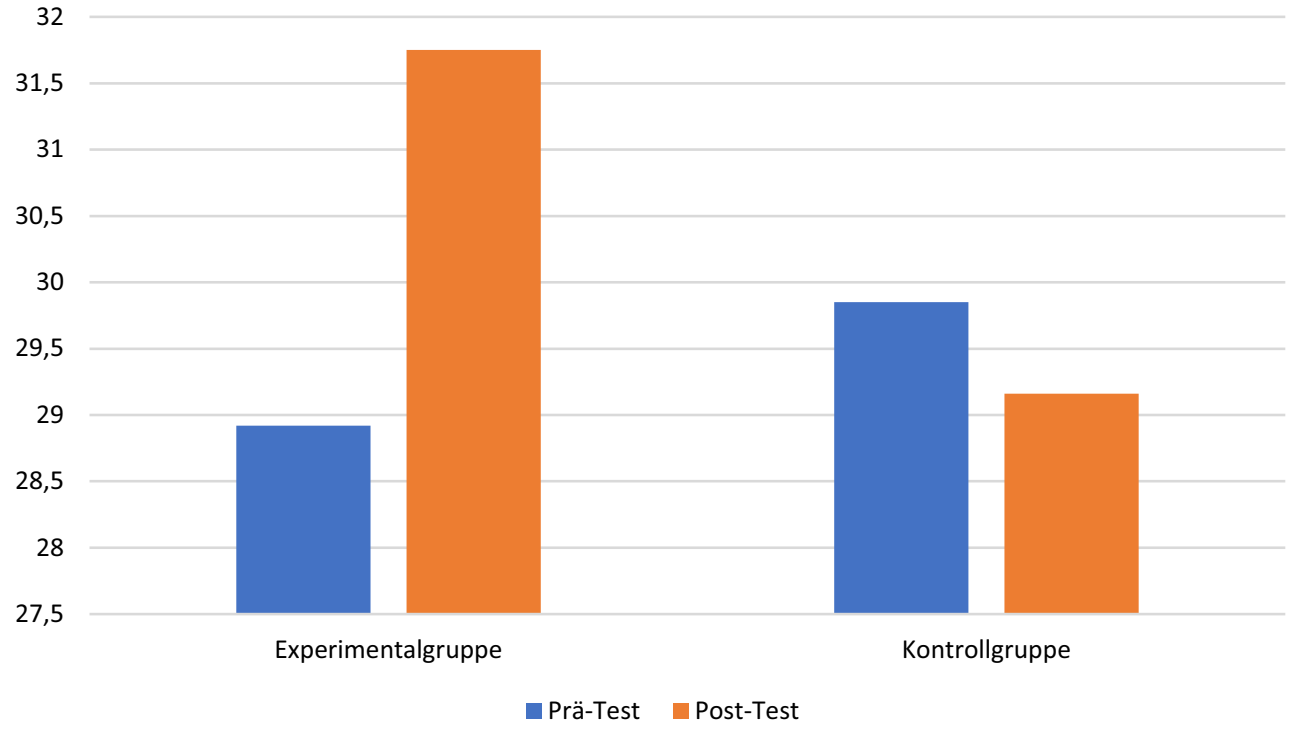

Zur Überprüfung der letzten Hypothese, innerhalb welcher ein Unterschied in der Selbstbewertung vor und nach einer Videoanalyse zu testen war, wurde aufgrund der fehlenden Normalverteilung der Wilcoxon-Test verwendet. Während 39 Personen nach der Videoanalyse eine positivere Selbstbewertung abgaben, war es bei sechs Teilnehmern andersherum. Sowohl der asymptotische als auch der exakte einseitig Signifikanzwert war hoch signifikant $(p<0,001)$. Mit einem z-Wert von $z=-4,85$ ergab sich eine Effektstärke von $d=0,67$; die nach Cohen (1992) als ein starker Effekt einzuordnen ist. Zusammenfassend kann auf Grund des signifikanten Ergebnisses auch die dritte Hypothese H3 beibehalten werden.

Die Prüfung eines Zusammenhangs zwischen Selbstbewertung und der Selbstwirksamkeitserwartung nach einem Coaching ergab, nach Ausschluss zweier Ausreißer, eine mittlere Korrelation von $r=0,42$. Somit ist zu erkennen, dass Personen mit einem hohen Wert der Selbstwirksamkeitserwartung nach dem Ende des Coachings einen höheren Wert in der Selbstbewertung aufwiesen.

Zusätzlich wurde mittels eines Wilcoxon-Tests geprüft, ob sich die Ergebnisse zwischen der Erst- und Zweitmessung innerhalb der Kontrollgruppe unterschieden. Der Unterschied zwischen diesen beiden Messungen ist jedoch auf einem Signifikanzniveau von $5 \%$ sowohl gemäß des einseitigen asymptotischen Signifikanzwertes von $p=0,05$ als auch dem exakten einseitigen Signifikanzwertes von $p=0,05$ nicht signifikant. Zudem wurde mittels eines MannWhitney-U-Tests zwischen der Experimental- und der Kontrollgruppe festgestellt, dass sich beide Gruppen bei der Erstmessung nicht signifikant unterschieden. Der Test ergab einen asymptotischen Signifikanzwertes von $p=0,12$. Somit gab es zum ersten Messzeitpunkt weder einen signifikanten Unterschied zwischen der Kontroll- und der Expe- rimentalgruppe, noch hat sich die Selbstwirksamkeitserwartung innerhalb der Kontrollgruppe vom ersten zum zweiten Messzeitpunkt signifikant verändert.

Mittels eines Mann-Whitney-U-Tests wurde zusätzlich sowohl bei der Erst- als auch bei der Zweitmessung überprüft, ob die Pferdeerfahrung der Teilnehmer einen Einfluss auf das Ergebnis hatte. Der zweiseitiger asymptotischer Signifikanzwert belief sich hierbei für den ersten Messzeitpunkt auf einen zweiseitigen, exakten Signifikanzwert $p=0,01$. Beim zweiten Messzeitpunkt ergab sich ein zweiseitiger exakter zweiseitiger Signifikanzwert von $p=0,14$. Es ergab sich damit für den ersten Messzeitpunkt ein hoch signifikanter Unterschied, während für den zweiten Messzeitpunkt kein signifikanter Unterschied zwischen pferdeerfahrenen und unerfahrenen Personen festgestellt werden konnte. Somit wird deutlich, dass sich die Gruppen bei der Erstmessung zunächst signifikant voneinander unterschieden, bei der zweiten Messung im Anschluss an das Coaching jedoch nicht mehr. Zusätzlich wurde bei Betrachtung der Mittelwerte der Selbstwirksamkeitserwartung von pferdeerfahrenen und unerfahrenen Personen festgestellt, dass bei beiden Personengruppen eine Steigerung der Selbstwirksamkeitserwartung vom ersten zum Messzeitpunkt festgestellt werden konnte. Die Differenz der Mittelwerte vom ersten zum zweiten Messzeitpunkt bei Personen mit Pferdeerfahrung lag bei 2,6 und bei Personen ohne Pferdeerfahrung bei 3,3. Die Selbstwirksamkeitserwartung veränderte sich bei Personen ohne Pferdeerfahrung demnach stärker als bei Personen mit Pferdeerfahrung. 


\section{Diskussion und Ausblick}

Aus den Ergebnissen dieser Untersuchung lässt sich schließen, dass sich sowohl die Selbstbewertung als auch die Selbstwirksamkeitserwartung durch ein pferdgestütztes Coaching positiv veränderten. Es konnte sowohl eine signifikante Steigerung der Selbstwirksamkeit vom Zeitpunkt von vor zu nach einem Coaching festgestellt werden als auch ein signifikanter Unterschied in der Selbstwirksamkeitserwartung zwischen Coachingteilnehmern und Personen, die kein Coaching erhielten. Bestätigend hierzu konnten auch Moen und Allgood (2009) sowie Baron und Morin (2010) feststellen, dass sich die Selbstwirksamkeit innerhalb eines Coachings erhöhen kann. Erklärt werden kann dies unter anderem dadurch, dass Erfolgserfahrungen als eines der stärksten Mittel gelten, um Selbstwirksamkeitserwartungen aufzubauen (Bandura 1994). Da das Pferd als Übungsobjekt für die Bewältigung von Aufgaben dient (Friesenhahn 2015a), kann dieses für den Teilnehmer das Sammeln von Erfolgserfahrungen ermöglichen. Zudem konnte in dieser Studie festgestellt werden, dass sich die Selbstbewertung innerhalb eines pferdegestützten Coachings ebenfalls verbessern kann.

Es bleibt weiterhin zu schlussfolgern, dass die verwendeten Methoden und das generelle Setting des pferdegestützten Coachings ausschlaggebend für diese Steigerung sind. Welche Maßnahmen oder Faktoren innerhalb des Coachings genau die signifikanten Veränderungen hervorgerufen haben, kann in dieser Untersuchung nicht eindeutig festgestellt werden. Den theoretischen Hintergrund betrachtend, können professionelle Feedbacks (Lindart 2016; Meyer 2009), gelenkte Selbstreflexionsmethoden, wie eine Videoanalyse (Finger-Hamborg 2005), sowie die generelle Begegnung und Zusammenarbeit mit dem Pferd (Friesenhahn 2015a) wichtige Wirkfaktoren des pferdegestützten Coachings darstellen, die sowohl eine positivere Selbstbewertung als auch eine Steigerung der Selbstwirksamkeitserwartung bedingen. Auch die Wirkung des Pferdes als sozialer Katalysator, Verhaltensspiegel und Übungspartner kann die Wirksamkeit des pferdegestützten Coachings positiv beeinflussen (Mc Nicholas und Collis 2000; Meyer 2009; Serad 2010). Es bleibt jedoch anzumerken, dass eine hohe Selbstwirksamkeitserwartung nicht das alleinige Ziel eines Coachings sein sollte. Vielmehr sollte sich der Klient differenziert mit sich selbst auseinandersetzen (Rauen 2011). Dabei können ihm sowohl das Pferd als verhaltensreflektierender Spiegel als auch die Methode der Videoanalyse helfen. Auch nach Finger-Hamborg (2005) ist die Videoanalyse eine zentrale und wirksame Methode im Coaching, die auch zu Verhaltensänderungen führen kann, da sie als Beweis für das Verhalten des Teilnehmers fungieren und damit Selbstreflexionsprozesse aktivieren kann (Rückle 2005). Selbstreflexion gilt dabei als der Prozess, bei dem man über sich selbst nachdenkt und das eigene Selbstkonzept hinterfragt. Da das Selbstkonzept als die Summe aller selbstbezogenen Gedanken und Gefühle definiert ist (Maddux 1995), kann die Selbstbewertung auch als Teilkomponente des Selbstkonzeptes verstanden werden. Eine ergebnisorientierte Selbstreflexion kann dem Klienten innerhalb des Coachings außerdem dazu dienen, sich über seine Bedürfnisse klar zu werden (Greif 2008). Somit liegt es nahe, dass eine selbstreflektierende Methode wie die Videoanalyse die Bewertung der eigenen Person verbessern kann. Das Ergebnis dieser Studie, dass eine signifikant positivere Selbstbewertung nach einer Videoanalyse zeigt, stützt diese These zusätzlich. Neben der Steigerung einer positiven Selbstbewertung ist die Förderung der Selbstwirksamkeitserwartung trotzdem ein wichtiges Ziel des Coachings, da durch eine hohe Selbstwirksamkeit Menschen geholfen werden kann, ihre Handlungsfähigkeit zu verbessern und ihren Optimismus zu stärken (Frontmüller et al. 2014; Göbel und Frese 1999; Röder 2009; Rosen 2004).

Die oben angebrachten Ergebnisse zeigen außerdem, dass sich Personen mit und Personen ohne Pferdeerfahrung bei der Messung vor dem Coaching zunächst signifikant voneinander unterschieden, jedoch bei der zweiten Messung im Anschluss an das Coaching nicht mehr. Von der Betrachtung der einzelnen Mittelwerte der beiden Gruppen vor und nach dem Coaching zu schließen, haben Menschen mit Pferdeerfahrung zunächst eine signifikant höhere Selbstwirksamkeitserwartung als Menschen ohne Pferdeerfahrung. Nach dem Coaching konnte bei beiden Gruppen eine höhere Selbstwirksamkeitserwartung gemessen werden, jedoch war der Unterschied zwischen beiden Gruppen nicht mehr signifikant. Somit wird deutlich, dass die Selbstwirksamkeitserwartung bei Menschen ohne Pferdeerfahrung mehr stieg als bei Menschen mit Pferdeerfahrung, wonach Personen ohne Pferdeerfahrung möglicherweise mehr von einem Coaching profitierten. Die hier generierten Ergebnisse lassen sich jedoch nur unter Betrachtung der methodischen Herangehensweise interpretieren. Zur Förderung der Vergleichbarkeit der Ergebnisse wurde bei der Durchführung jedoch stets darauf geachtet, das Treatment möglichst konsistent zu halten. So durchliefen alle Coachingteilnehmer einen ähnlichen Ablauf, sofern dies in einem Klienten zentrierten Ansatz möglich war. Die Entscheidung für zwei Messzeitpunkte diente zudem der Erhöhung der internen Validität des quasi-experimentellen Versuchsdesigns (Döring und Bortz 2016). Den Messzeitpunkt auf einen zeitlichen Abstand von einer Woche zu legen, galt der Kontrolle möglicher Störeffekte. So sollten hiermit Carry-Over-Effekte, wie beispielsweise der Erinnerungseffekt oder auch das Priming auf bestimmte Aspekte der Erstmessung, gemindert werden (Heller 2012). Eine Limitation liegt hier jedoch in dem kurzen Zeitraum von nur einer Woche und zudem darin, dass lediglich zwei 
Gruppen miteinander verglichen wurde. Eine Gruppe, die ein Coaching ohne Pferd erhielt, gab es nicht. Beim Online-Fragebogen stellte sich heraus, dass die Abbruchquote (Drop-out) sehr hoch war (82\%). Generell sind hohe Abbruchquoten einem zweiten Erhebungszeitpunkt geschuldet (Rack und Christophersen 2009). Auch bei dieser Untersuchung fehlte zur Auswertung meist der zweite Teil des Fragebogens. Dies kann dem Fakt geschuldet sein, dass die Teilnehmer selbstständig daran denken mussten, den zweiten Teil des Fragebogens nach einer Woche erneut zu beantworten. Hierdurch ist es auch denkbar, dass die Stichprobe besonders viele Versuchspersonen beinhaltet, die sehr gewissenhaft sind oder dem Thema dieser Untersuchung sehr zugewandt, was eine weitere Limitation darstellen könnte. Es ist darüber hinaus nicht bekannt, was die Teilnehmer in der Zwischenzeit zwischen der Beantwortung der Fragebögen erlebt haben. Das unkontrollierbare, zwischenzeitliche Geschehen wird generell als Störfaktor bezeichnet (Rack und Christophersen 2009). Hier können zufällige Lebensereignisse, die auf die Selbstwirksamkeitserwartung Einfluss nehmen, das Ergebnis beeinflusst haben. Auch bei der Messung der Selbstbewertung lag zwischen den beiden Messzeitpunkten nicht nur die Videoanalyse, sondern beispielsweise auch das Feedback des Coaches. Somit könnte auch dies einen Einfluss auf die Ergebnisse gehabt haben, was ebenfalls als mögliche Limitation zu betrachten ist. Die Skala zur Allgemeinen Selbstwirksamkeitserwartung von Schwarzer und Jerusalem (1999) betrachtend, ist festzuhalten, dass hier keine Kontrolle der sozialen Erwünschtheit gewährleistet werden kann. Auf Grund der Tatsache, dass die meisten Werte in höheren Bereichen lagen, ist davon auszugehen, dass die soziale Erwünschtheit hier einen Störeffekt darstellt, beispielsweise weil Personen sich keine schlechte Lösungskompetenz eingestehen wollen (Hussy et al. 2013). Um diesen Fehler jedoch möglichst zu minimieren, wurden die Fragebögen komplett anonym erhoben (Hussy et al. 2013).

In nachfolgenden Experimenten könnte untersucht werden, ob bestimmte Methoden eines pferdegestützten Coachings einen besonders hohen oder niedrigen Einfluss auf die Selbstwirksamkeitserwartung haben. Nicht nur die Methoden, sondern auch das Pferd in seiner Rolle im Coaching könnte einen Wirkfaktor für die Steigerung der Selbstwirksamkeit darstellen. Inwiefern die Steigerung der Selbstwirksamkeitserwartung hierbei dem Einsatz des Pferdes zuzuschreiben ist und wie stark sich eine Förderung der Selbstwirksamkeit im Vergleich zu anderen Coachingformen unterscheidet, bleibt offen. In einer fortführenden Vergleichsstudie verschiedener Coachingformen mit dem pferdegestützten Coaching könnte dies ermittelt werden. Hier sollte ein besonderer Fokus auf eine zusätzliche Interventionsgruppe ohne Pferd gelegt werden, um den Vergleich zwischen Coachings mit und ohne Pferd zu untersuchen.
Den Ergebnissen von Finn et al. (2007) zufolge kann die Selbstwirksamkeitserwartung im Anschluss an ein Coaching auch nach sechs Monaten noch steigen. Eine solche Langzeitwirkung kann hier nicht nachgewiesen werden, da lediglich zwei Messzeitpunkte (vor und nach dem Coaching) untersucht wurden. Ob der Effekt der Steigerung der Selbstwirksamkeit durch ein pferdegestütztes Coaching auch nach mehreren Monaten noch sichtbar ist, bleibt daher in einer Langzeitstudie zu untersuchen. In der Erhebung der Normstichprobe von Hinz et al. (2006) zeigte sich zudem, dass Männer eine höhere Selbstwirksamkeitserwartung aufweisen als Frauen. Hier könnte eine weitere Untersuchung der Geschlechterunterschiede bezüglich der Selbstwirksamkeit interessant sein, da dies in der Praxis innerhalb eines Coachings beachtet werden könnte. Weiterhin konnte festgestellt werden, dass die Selbstbewertung nach einer Videoanalyse positiver ausfiel als davor. Ob die Videoanalyse alleiniger Wirkfaktor hierfür war, kann nicht eindeutig festgemacht werden, da auch das Feedback des Coaches zwischen beiden Messzeitpunkten lag.

Der hier erbrachte Nachweis einer Steigerung der Selbstwirksamkeit sowie einer positiveren Selbstbewertung innerhalb eines pferdegestützten Coachings sind zudem ein weiteres wissenschaftlich fundiertes Pro-Argument für ein pferdegestütztes Coaching. Trotz des in dieser Studie generierten Erkenntnisgewinns bleiben jedoch noch viele Fragen auf dem bislang wenig erforschten Gebiet des pferdegestützten Coachings offen. Daher sollten weitere empirische Untersuchungen zu dieser Thematik folgen.

Interessenkonflikt Prof. Dr. Kathrin Schütz arbeitet als Coach, bei Julia Steinhoff liegt kein Interessenkonflikt vor.

Open Access Dieser Artikel wird unter der Creative Commons Namensnennung 4.0 International Lizenz (http://creativecommons.org/ licenses/by/4.0/deed.de) veröffentlicht, welche die Nutzung, Vervielfältigung, Bearbeitung, Verbreitung und Wiedergabe in jeglichem Medium und Format erlaubt, sofern Sie den/die ursprünglichen Autor(en) und die Quelle ordnungsgemäß nennen, einen Link zur Creative Commons Lizenz beifügen und angeben, ob Änderungen vorgenommen wurden.

Publisher's Note Springer Nature remains neutral with regard to jurisdictional claims in published maps and institutional affiliations.

\section{Literatur}

Bandura, A. (1994). Self-efficacy. In V. S. Ramachaudran (Hrsg.), Encyclopedia of human behavior (S. 71-81). New York: Academic Press.

Bandura, A. (1997). Self-efficacy: the exercise of control. New York: Freeman.

Baron, L., \& Morin, L. (2010). The impact of executive coaching on self-efficacy related to management soft-skills. Leadership \& Organization. Development Journal, 31, 18-38.

Boeckle, M., Katzlinger, M., Lackner, O., Barborik, M., Püspök, J., Leitner, A., et al. (2016). Der Einfluss von Selbstwirksamkeit und intentionalem Verhalten auf den Therapieerfolg chronischer 
Schmerzpatienten. Krems: Donau-Universität. Wissenschaftliches Poster

Cohen, J. (1992). A power primer. Psychological Bulletin, 112, $155-159$.

Dampsey, E. (2017). The effects of equine-assisted psychotherapy on emotion regulation: self-efficacy and self-awareness as potential mediators. Fielding: Graduate University. Unpublished Dissertation

Döring, N., \& Bortz, J. (2016). Untersuchungsdesign. In N. Döring \& J. Bortz (Hrsg.), Forschungsmethoden und Evaluation in den Sozialund Humanwissenschaften (S. 181-186). Heidelberg: Springer.

Ewing-Chow, S. A. (2014). Truth tellers: coaching with horses. https:// coachcampus.com/coach-portfolios/research-papers/suzanne-aewing-chow-truth-tellers-coaching-with-horses/. Zugegriffen: 18. Juni 2018.

Finger-Hamborg, A. (2005). Einzel-Coaching mit Schichtleitern Ein Erfahrungsbericht. In C. Rauen (Hrsg.), Handbuch Coaching (S. 369-390). Göttingen: Hogrefe.

Finn, F. A., Mason, C. M., \& Bradley, L. M. (2007). Doing well with executive coaching: psychological and behavioral impacts. In G. Solomon (Hrsg.), Proceedings academy of management 2007 annual meeting proceedings: doing well by doing good (S. 1-34). Philadelphia: Academy of Management.

Friedmann, E., Katcher, A.H., Thomas, S. A., Lynch, J.J., \& Messent, P. R. (1983). Social interaction and blood pressure: influence of animal companions. Journal of Nervous and Mental Disease, $171(8), 461-464$.

Friesenhahn, J. (2015a). Coaching mit Pferden. Coaching Magazin, 4, 45-47. Eine Ergänzung zu Prof. Dr. Kannings Beitrag im Coaching-Magazin 4/2014.

Friesenhahn, J. (2015b). Unter sechs Augen. Die Wirkung von Pferden im Coaching. Coaching-Magazin, 4, 48-53.

Frontmüller, R., Katzgraber, D., \& Schalko, K. (2014). Selbstwirksamkeitserwartungen und Burnout-Gefährdung - Eine empirische Studie zum Zusammenhang zwischen den Selbstwirksamkeitserwartungen und der Burnout-Gefährdung von Handelsakademieschülern/-schülerinnen. Wissenschaftsplus, 32, 1-5.

Geddes, J.H. (2010). Self-efficacy and equine assisted therapy: A single subject study. http://commons.pacificu.edu/spp/202. Zugegriffen: 18. Juni 2018

Göbel, S., \& Frese, M. (1999). Persönlichkeit, Strategien und Erfolg bei Kleinunternehmern. In K. Moser, B. Batinic \& J. Zempel (Hrsg.), Unternehmerisch erfolgreiches Handeln (S. 93-113). Göttingen: Verlag für Angewandte Psychologie.

Gomolla, A. (2014). Pferde haben Spiegelfunktion. Deutsches Ärzteblatt, 12(8), 356-357.

Gomolla, A., Keser, B., Haag, A., Irschei, J., \& Fürst, J. (2011). Das Pferd als Spiegel des Menschen - Reaktion auf Körperspannung und -haltung. http://www.great-horses.org/media/Downloads/ Spiegelfunktion\%20-\%20WEB.pdf. Zugegriffen: 14. Juni 2018.

Grawe, K. (1998). Psychologische Therapie. Göttingen: Hogrefe.

Greif, S. (2008). Coaching und erlebnisorientierte Selbstreflexion. Göttingen: Hogrefe.

Greif, S., \& Rauen, C. (2017). Coaching. In M. A. Wirtz (Hrsg.), Lexikon der Psychologie (S. 344). Göttingen: Hogrefe.

Greiffenhagen, S., \& Buck-Werner, O.N. (2011). Tiere als Therapie. Nerdlen: Kynos.

Heller, J. (2012). Experimentelle Psychologie. Eine Einführung. München: Oldenbourg.

Heß, T., \& Roth, W.L. (2001). Professionelles Coaching. Heidelberg: Asanger.

Hinz, A., Schumacher, J., Albani, C., Schmid, G., \& Brähler, E. (2006). Bevölkerungsrepräsentative Normierung der Skala zur Allgemeinen Selbstwirksamkeitserwartung. Diagnostica, 35, 26-32.

Hussy, W., Schreier, M., \& Echterhoff, G. (2013). Forschungsmethoden in Psychologie und Sozialwissenschaften für Bachelor. Heidelberg: Springer.
Jerusalem, M. (1990). Persönliche Ressourcen, Vulnerabilität und Stresserleben. Göttingen: Hogrefe.

Jerusalem, M. (1991). Allgemeine Selbstwirksamkeit und differentielle Stressprozesse. Psychologische Beiträge, 33, 388-406.

Jerusalem, M. (2005). Selbstwirksamkeit. In H. Weber \& T. Rammsayer (Hrsg.), Handbuch der Persönlichkeitspsychologie und Differentiellen Psychologie (S. 438-445). Göttingen: Hogrefe.

Konir, G. (2012). Pferdegestütztes Coaching. Menschliche Potenzialentwicklung durch tierische Hilfe. Norderstedt: Books on Demand.

Krüger, K., Flauger, B., Farmer, K., \& Maros, K. (2011). Horses (Equus caballus) use human local enhancement cues and adjust to human Attention. Animal Cognition, 14, 187-201.

Lang, U.E., Jansen, J. B., Wertenauer, F., Gallinat, J., \& Rapp, M. A. (2010). Reduced anxiety during dog assisted interviews in acute schizophrenic patients. European Journal of Integrative Medicine, 2, 123-127.

Lindart, M. (2016). Was Coaching wirksam macht. Wiesbaden: Springer.

MacDonald, P. M. (2004). The effects of equine-facilitated therapy with at-risk adolescents: A summary of empirical research across multiple centers and programs. The Center for the Interaction of Animals and Society (CIAS). Philadelphia: University of Pennsylvania School of Veterinary Medicine.

Maddux, J.E. (1995). Self-efficacy theory: An introduction. In J.E. Maddux (Hrsg.), Self-efficacy, adaption, and adjustment (S. 3-33) New York: Plenum Press.

Mc Burney, D.H., \& White, T.L. (2010). Research methods. Wadsworth: Belmont.

Mc Nicholas, J., \& Collis, G. M. (2000). Dogs as catalysts for social interactions: robustness of the effect. British Journal of Psychology, 91, 61-70.

Meyer, S. (2009). Pferde als Medium im Coaching: natürlich, ehrlich und nachhaltig! Coaching-Magazin, 4, 42.

Mitteregger, S. B. (2011). Evaluierung einer pferdegestützten psychologischen Therapie im Rahmen einer stationären psychosomatischen Rehabilitation. Graz: Medizinische Universität. Unveröffentlichte Dissertation

Moen, F., \& Allgood, E. (2009). Coaching and the effect on self-efficacy. Organization Development Journal, 27, 69-81.

Nakamura, K., Takimoto-Inose, A., \& Hasegawa, T. (2018). Crossmodal perception of human emotion in domestic horses (Equus caballus). Scientific Reports, 8, 8660. https://doi.org/10.1038/ s41598-018-26892-6.

Otterstedt, C. (2001). Tiere als therapeutische Begleiter. Stuttgart: Kosmos.

Rack, O., \& Christophersen, T. (2009). Experimente. In S. Albers, D. Klapper, U. Konradt, A. Walter \& J. Wolf (Hrsg.), Methodik der empirischen Forschung (S. 17-32). Wiesbaden: Springer.

Rauen, C. (2005). Varianten des Coachings im Personalentwicklungsbereich. In C. Rauen (Hrsg.), Handbuch Coaching (S. 111-136). Göttingen: Hogrefe.

Rauen, C. (2011). Coaching-Newsletter April 2011. https://www. coaching-newsletter.de/archiv/2011/2011-04.html. Zugegriffen: 9. Juli 2018.

Rauen, C. (2014). Coaching. Göttingen: Hogrefe.

Röder, B. (2009). Selbstwirksamkeitsförderung durch Motivierung von Schülern. Berlin: Freie Universität Berlin. Unveröffentlichte Dissertation

Rosen, M. (2004). Zum Einfluss beruflicher Selbstwirksamkeitserwartungen und persönlicher Ziele auf beruflichen Erfolg: Eine empirische Untersuchung bei Arbeitnehmerinnen und Arbeitnehmern in einem mittelständischen Unternehmen. Berlin: Freie Universität Berlin. Unveröffentlichte Diplomarbeit.

Rückle, H. (2005). Gruppen-Coaching. In C. Rauen (Hrsg.), Handbuch Coaching (S. 183-198). Göttingen: Hogrefe. 
Ruholl, S. (2007). Selbstwirksamkeit als Indikator für psychische Störungen - Satus und Verlauf. Aachen: Rheinisch-Westfälischen Technischen Hochschule. Unveröffentlichte Dissertation

Satow, L., \& Bäßler, J. (1998). Selbstwirksamkeit und körperliches Befinden Jugendlicher. Unterrichtswissenschaft, 26, 127-139.

Schreyögg, A. (2003). Coaching. Frankfurt am Main: Campus.

Schunk, D.H. (1995). Self-efficacy and education and instruction. In J.E. Maddux (Hrsg.), Self-efficacy, adaptation, and adjustment. Theory, research, and application (S. 281-303). New York: Plenum.

Schütz, K., Rötters, A., \& Oebel, L. (2018). Können Pferde als CoTrainer agieren? Individuelle Reaktionen von Pferden in der Persönlichkeitsentwicklung auf unterschiedliche Klienten. Tiergestützte Therapie, Pädagogik \& Fördermaßnahmen, 1/2018, 22-26.

Schütz, K. (2018). Pferde, Forschung \& Psychologie. Wissenschaftliche Befunde zu Fähigkeiten von Pferden und deren Wirkung auf Menschen. Kindle.

Schwarzer, R. (1994). Optimistische Kompetenzerwartung: Zur Erfassung einer personalen Bewältigungsressource. Diagnostica, 40, $105-123$.
Schwarzer, R., \& Jerusalem, M. (1999). Skalen zur Erfassung von Lehrer- und Schülermerkmalen. Dokumentation der psychometrischen Verfahren im Rahmen der wissenschaftlichen Begleitung des Modellversuchs Selbstwirksame Schulen. Berlin: Freie Universität Berlin.

Schwarzer, R., \& Jerusalem, M. (2002). Das Konzept der Selbstwirksamkeit. Zeitschrift für Pädagogik, 44, 28-53.

Schwarzer, R., Mueller, J., \& Greenglass, E. (1999). Assessment of perceived general self-efficacy on the Internet: Data collection in cyberspace. Anxiety, Stress, and Coping, 12, 145-161.

Serad, L. (2010). Aspects of using animal-assisted interventions in a coaching model. Philadelphia: University of Pennsylvania. Unpublished master thesis

Wakkee, I., Elfring, T., \& Monaghan, S. (2010). Creating entrepreneurial employees in traditional service sectors - the role of coaching and self-efficiacy. International Journal of Entrepreneurial Management, 6, 1-21.

Warner, L.M. (2017). Selbstwirksamkeitserwartung. In M.A. Wirtz (Hrsg.), Dorsch Lexikon der Psychologie (S. 1527). Göttingen: Hogrefe. 